\title{
Surface-Enhanced Raman Difference between Human Insulin and Insulin Lispro Detected with Adaptive Nanostructures
}

\author{
Vladimir P. Drachev, ${ }^{* \dagger}{ }^{\dagger}$ Mark D. Thoreson,${ }^{\dagger}$ Eldar N. Khaliullin, ${ }^{\dagger}$ V. Jo Davisson, ${ }^{\ddagger}$ and \\ Vladimir M. Shalaev ${ }^{\dagger}$ \\ School of Electrical and Computer Engineering and School of Pharmacy and Pharmaceutical Sciences, \\ Purdue University, West Lafayette, Indiana 47907
}

Received: June 23, 2004; In Final Form: September 5, 2004

\begin{abstract}
A protein sensor based on nanostructured adaptive silver films (ASFs) is developed for soft protein adsorption and detection with surface-enhanced Raman scattering (SERS). Under protein deposition, the ASFs modify their local nanostructure so that the conformational state of proteins is preserved and SERS is optimized. The sensor is used to examine differences in Raman spectra of two insulin isomers, human insulin and its analogue insulin lispro. These two insulins differ only in the interchange of two neighboring amino acids; specifically, the propyl-lysyl sequence at the C-terminus of the B-chain in insulin lispro is inverted as compared to human insulin. This switch leads to conformational changes at the $\mathrm{C}$ - and $\mathrm{N}$-termini and has an important clinical effect for diabetes treatment. The difference in SERS spectra for the two insulins was detected at a submonolayer density, $80 \mathrm{fmol} / \mathrm{mm}^{2}$, with only $25 \mathrm{amol}$ in the probed area, and macroscopic enhancement factor $3 \times 10^{6}$. The performed studies indicate that SERS detection using ASFs can lead to efficient proteomic sensing technology.
\end{abstract}

\section{Introduction}

Protein sensing using Raman spectroscopy provides important structural information on conformational changes. Changes between native and denatured insulin in the solid form as well as spectral features of proinsulin and insulin fibrils were studied previously. ${ }^{1-3}$ Signatures of allosteric conformation changes in hexameric insulin have been assessed using Raman difference spectroscopy. ${ }^{4}$

Surface-enhanced Raman scattering (SERS) provides greater sensitivity than conventional Raman spectroscopy, ${ }^{5-7}$ and it is becoming a powerful technique in probing biological molecules adsorbed on a metal surface. ${ }^{8-16}$ SERS is sensitive to molecule orientation and the distance from the metal surface. ${ }^{17}$ Therefore, SERS spectroscopy can be particularly efficient in detecting conformational changes and structural differences related to preferred orientations of proteins with respect to the metal surface. It is possible to obtain high-quality SERS spectra at submonolayer molecular coverage as a result of the large surface enhancements. The SERS mechanism is originated in part from the large local electromagnetic fields caused by resonant surface plasmons that can be optically excited at certain wavelengths for metal particles of different shapes or compact groups of particles. ${ }^{18-25}$ The plasmon resonances can be excited in a broad spectral range for aggregates of interacting particles often structured as fractals. ${ }^{26}$ In addition, metal nanostructures and molecules can form charge-transfer complexes, providing further enhancement for SERS. ${ }^{27-33}$ The resultant enhancement depends critically on the nanostructure morphology, ${ }^{26,34-40}$ and it can be as high as $10^{5}-10^{8}$ for the macroscopic area averaged signal and $10^{10}-10^{15}$ within the local resonant nanostructures.

Among SERS active substrates, evaporated nanostructured metal films are well-suited for SERS mechanism studies and

* Corresponding author. E-mail: vdrachev@ecn.purdue.edu.

School of Electrical and Computer Engineering.

$\doteqdot$ School of Pharmacy and Pharmaceutical Sciences. have a high potential for applications. ${ }^{18-24,28,41-47}$ A variety of structures, aggregated films, ${ }^{19}$ islands of different shapes, ${ }^{18,19,21-24}$ and semicontinuous films near the percolation threshold ${ }^{45-47}$ are found to be appropriate for SERS. The effect of deposition rate, mass thickness dependence, and thermal annealing were studied in detail. ${ }^{22,41-44}$ The concepts of enhanced local field in aggregated particle films based on a Maxwell-Garnett approach were applied to SERS in the 1980s. ${ }^{18,19,21-23}$ These theories account for particle shape and particle interaction and emphasize that the large local fields can only be the result of large field fluctuations. ${ }^{20}$ The metal islands can serve as resonant enhancers for both the incident and scattered radiation, as it was shown for isolated particle ${ }^{20}$ and then generalized for smallparticle composite ${ }^{26}$ and percolation films. ${ }^{46,47}$ Both experiment ${ }^{19,22}$ and theory ${ }^{21,22}$ reveal a correlation between electromagnetic enhancement and the effective dielectric properties of metal-dielectric films. Namely, the enhanced Raman intensity is proportional to the product of absorption at laser and scattered wavelength $A_{\mathrm{l}} A_{\mathrm{s}},{ }^{19,21-23}$ which gives guidance for preliminary substrate parameter selection. The macroscopic enhancement is proportional to the square of the product of the plasma frequency and the relaxation time $\left(\omega_{\mathrm{p}} \tau_{\mathrm{eff}}\right)^{2}$ for island films ${ }^{22}$ and to the third power of the product for 2-D percolation films. ${ }^{46,47}$ Using data for $\mathrm{Ag}\left(\omega_{\mathrm{p}}=9.1 \mathrm{eV}, \tau_{\mathrm{eff}}{ }^{-1}=0.021 \mathrm{eV}\right)$, $\mathrm{Au}\left(\omega_{\mathrm{p}}=9.0 \mathrm{eV}, \tau_{\mathrm{eff}}{ }^{-1}=0.066 \mathrm{eV}\right)$, and $\mathrm{Cu}\left(\omega_{\mathrm{p}}=8.8 \mathrm{eV}\right.$, $\left.\tau_{\text {eff }}{ }^{-1}=0.09 \mathrm{eV}\right),{ }^{48,49}$ one can see that an average electromagnetic enhancement of the Raman scattering should be much larger for $\mathrm{Ag}$ than for $\mathrm{Au}$ or $\mathrm{Cu}$.

We employ electron beam evaporated Ag films for protein sensing with SERS and introduce one more factor into consideration, which is a restructuring of island film under protein deposition. Such kinds of evaporated metal films, which allow restructuring, will be called adaptive herein. One can provide certain parameters of evaporation and protein deposition to make films adaptive. This feature of evaporated films plays an important role for the following reasons. Along with the 


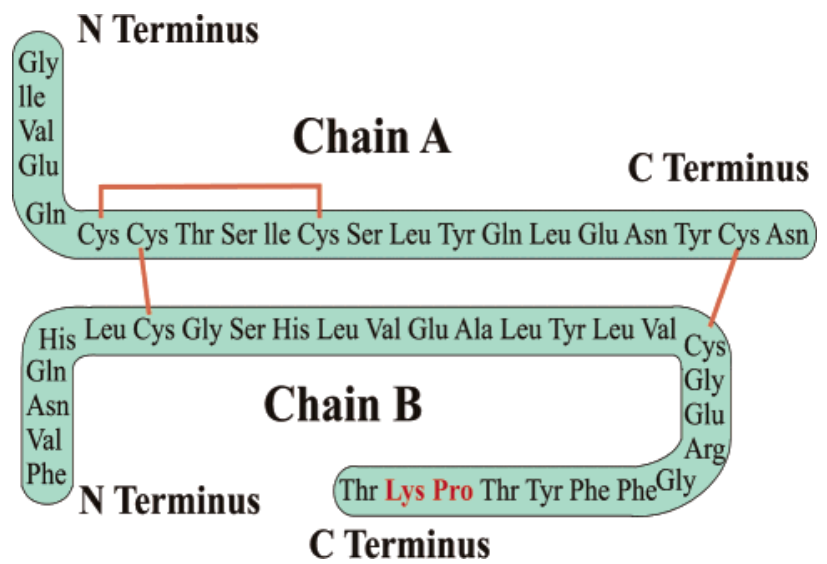

Figure 1. Two chains of 51 amino acids in human insulin.

advantages provided by SERS, there are two problems that need to be addressed. First, direct adsorption of proteins on a metal surface often leads to their significant structural (denaturation) and/or functional changes. , $^{12,50,51}$ This means that not any substrate is biocompatible. Second, any particular metal nanostructure with a fixed morphology cannot perfectly match different proteins and provide the optimal SERS in all cases. In a particular example, SERS spectra of bovine insulin adsorbed on silver electrode reveal dissimilar features with Raman spectrum of insulin aqueous solution. ${ }^{52}$ Our approach based on adaptive metal films allows fine nanostructure rearrangement under protein deposition that makes it possible to address both problems simultaneously, namely, to adsorb proteins on the metal surface without significant structural changes (softadsorption) and to optimize the SERS signal for given set of particles. In particular, we demonstrate that adaptive nanostructured films can be used for the observation of differences in SERS spectra of recombinant human insulin and insulin lispro at submonolayer protein surface densities. Since human insulin and its analogue have the same set of side chains, and differ only in conformational states, the observed difference indicates the preservation of the conformational state with the use of adaptive metal substrates. Raman difference spectroscopy is a general method of probing protein structure for comparison between closely related proteins. ${ }^{53} \mathrm{We}$ extend this method to SERS in order to study the spectral features of insulin conformation from two insulin analogues with the same set of amino acids.

Human Insulin and Insulin Lispro. Insulin is composed of two peptide chains referred to as A- and B-chains (see Figure 1). The two chains are linked together by two disulfide bonds, and an additional disulfide is formed within the A-chain. The A-chain consists of 21 amino acids, while the B-chain has 30 amino acids. Monomeric insulin is the active form of the hormone. Insulin exists as a monomer in solution at neutral $\mathrm{pH}$ and at physiological concentrations (about $1 \mathrm{ng} / \mathrm{mL}$ ). Hydrogen bonding between $\mathrm{C}$-termini of the $\mathrm{B}$-chains promotes the formation of dimers; antiparallel-pleated-sheet interactions further stabilize the insulin dimers. Oligomerization to the less soluble hexamer state is promoted by zinc ions (see Figure 2), which has significant consequences on the solubility of the protein. The clinical efficacy of the hexameric insulin is reduced due to the delayed absorption rate.

The problem of insulin oligomerization has stimulated development of a number of recombinant insulin analogues. The first of these molecules, which is called insulin lispro, is engineered so that lysine and proline residues on the $\mathrm{C}$-terminus of the B-chain are interchanged in their positions (see Figure

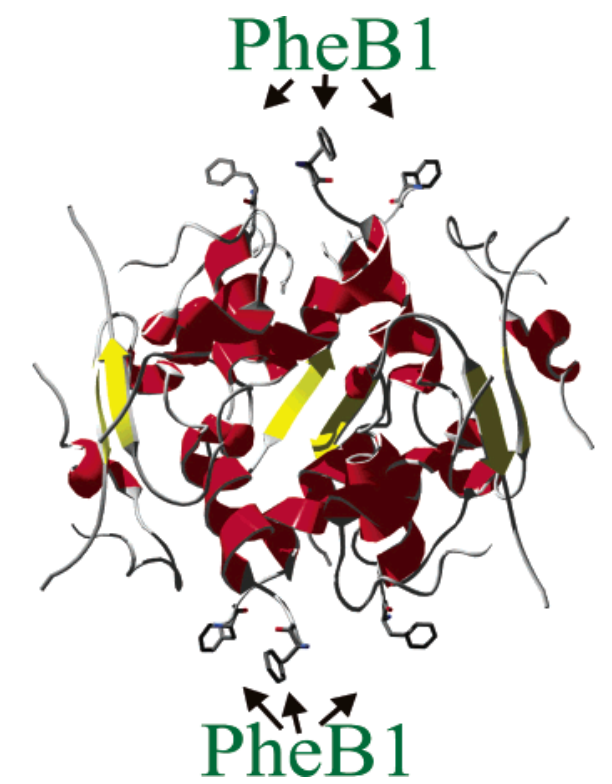

Figure 2. Human insulin hexamer (Protein Data Base). N-terminals (PheB1) of B-chains are exposed to the hexamer surface (see the top and bottom parts).

1). Thus, lispro is $\operatorname{Lys}(\mathrm{B} 28) \operatorname{Pro}(\mathrm{B} 29)$ and is an isomeric human insulin analogue, having the identical chemical composition and molecular weight as human insulin. The lispro modification minimizes the tendency to form dimers and hexamers but does not alter receptor binding. As a result, insulin lispro is a rapidly acting, parenteral blood glucose-lowering agent. The allosteric properties of insulin are critically important in design of insulin formulations with the required chemical and physical stability for use in the treatment of diabetes. ${ }^{54}$

Adaptive Silver Film Characterization. Our SERS protein sensor is based on nanostructured adaptive silver films (ASFs) formed on a dielectric substrate under vacuum evaporation with an electron beam (see details below in ref 55). In the deposition process, small isolated metal granules are formed first; as the metal coverage increases, the granules coalesce, resulting in various aggregates of particles. The film mass thickness and deposition rate were monitored with a quartz crystal oscillator. The glass slides were covered first by a sublayer of $10 \mathrm{~nm}$ of $\mathrm{SiO}_{2}$ followed by an $8 \mathrm{~nm} \mathrm{Ag}$ layer deposited at a rate of 0.05 $\mathrm{nm} / \mathrm{s}$. The substrates were used within 1 month after preparation.

The effect of restructuring under insulin deposition reveals in the extinction spectra and field-emission scanning microscope (FE SEM) images.

Typical UV-vis absorbance and reflectance spectra of initial substrate are similar and have a maximum at $570 \mathrm{~nm}$ and a broad wing in the long wavelengths. Reflection is greater than absorption by a factor of 1.4 (both are expressed in percent). The color of the film and the extinction spectrum are changed after insulin deposition, as is shown in Figure 3. The spot is deposited from a $3 \mu \mathrm{L}$ drop of $1 \mu \mathrm{M}$ insulin in a $0.1 \mathrm{mM} \mathrm{HCl}$ water solution and then dried. The spectrum inside the spot shows a blue-shifted maximum, reduced slope of the longwavelength wing, and reduced extinction integrated over the available spectral range.

In Figure 4 we show representative field-emission scanning electron microscope (FE SEM) images of an $8 \mathrm{~nm}$ ASF taken outside (top) and inside (middle) of a protein spot. They consist of nanoparticles and their aggregates. The particle size is distributed in a range from 30 to $100 \mathrm{~nm}$ with an aspect ratio between 0.5 and 1 (a typical value is $0.8-0.9$ ). The large-sized particles have an internal structure and more complicated shape. 

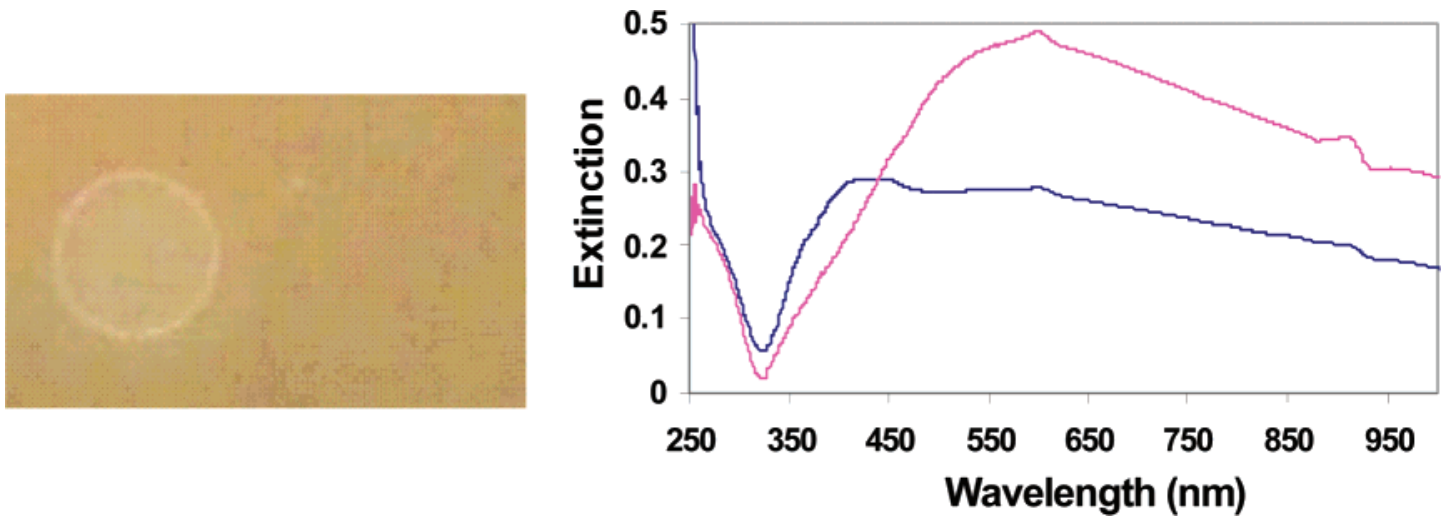

Figure 3. Insulin spot on adaptive substrate (a, left) and extinction spectra outside (red) and inside the spot (blue) (b, right).
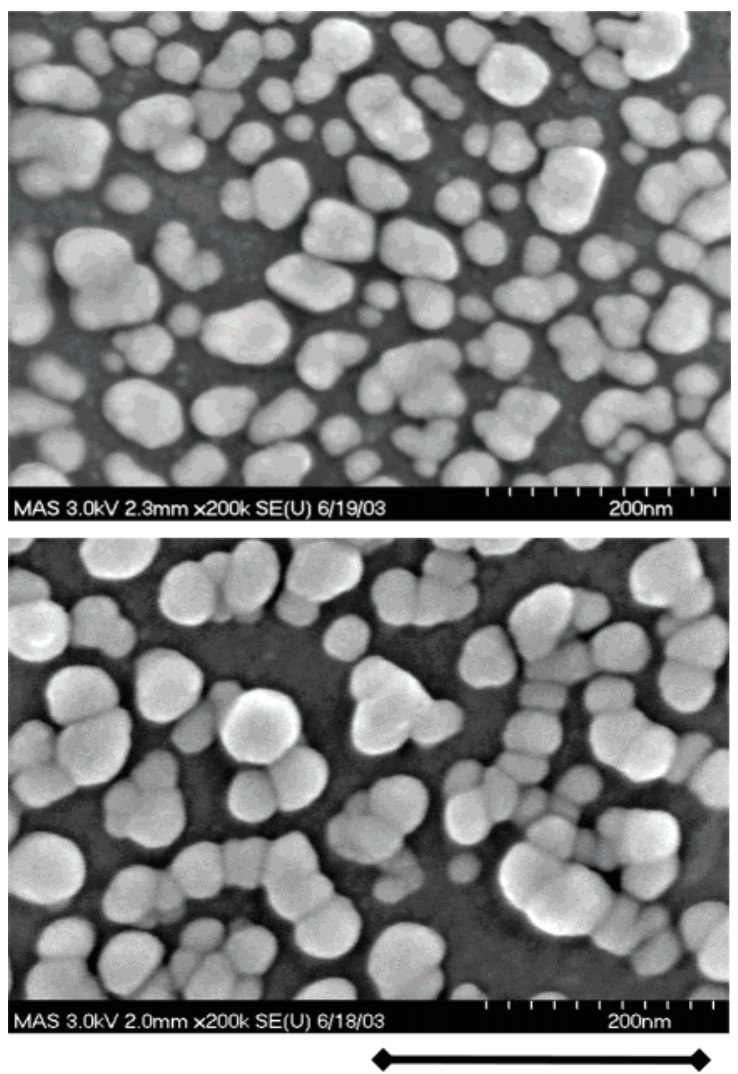

$300 \mathrm{~nm}$

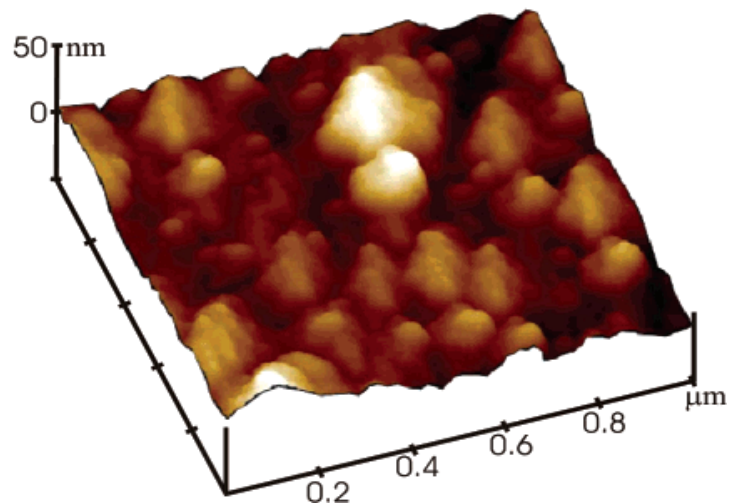

Figure 4. FE-SEM images of the ASF of $8 \mathrm{~nm}$ mass thickness outside (top) and inside (middle) a protein spot; AFM image of the $8 \mathrm{~nm} \mathrm{ASF}$ (bottom).

The figure clearly shows that the film experiences local restructuring in the protein spot, where groups of closely spaced metal nanoparticles are formed, as opposed to rather disintegrated particles outside the protein spot. A filling factor calculated as the ratio of an area covered with metal (white) to the total area is reduced from an initial value of 0.65 to 0.54 inside the spot.

A typical AFM height profile is also shown in the bottom of Figure 4. We note that the ASFs are uniform on the scale of micrometers and higher so that they are very homogeneous within a laser spot. The root mean square (RMS) deviation from the average height as defined with the roughness analysis of the AFM images is about $7 \mathrm{~nm}$, and the maximum height is about $30 \mathrm{~nm}$, while RMS deviation was only about $5 \mathrm{~nm}$ inside the protein spot.

Decreases in the integrated extinction and in the metal filling factor both suggest a decrease of the silver mass thickness caused by deposition. Varying insulin and $\mathrm{HCl}$ concentration, we found that both factors are important. Deposition of a lower insulin concentration by a factor of 10-20 results in an almost transparent spot without metal particles. Most likely the acidic solvent $(0.1 \mathrm{mM} \mathrm{HCl})$ dissolves particles, leaving just silver salt on the substrate. This process may affect the interface between Ag particles and silica and decrease adhesion or even remove particles in solution. Deposition of insulin solution without $\mathrm{HCl}$ reveals no visible changes of the film surface. ${ }^{56}$ Although the mechanism of the restructuring requires further study, one can conclude that the structure modification is a result of competition between dissolving of the metal surface and its stabilization by proteins.

The conventional approach suggests that the metal structures should have the highest possible adhesion to the substrate. The observed restructuring implies that some optimal adhesion is realized when metal structures adhere to the substrate well enough to remain on the substrate while still preserving some limited ability to move in the plane of the film so that soft protein adsorption, causing no protein denaturation, is possible. In parallel, the SERS signal is also improved under such restructuring; this is because proteins "glue" neighbor metal particles so that the gaps between the particles become smaller and are just enough to accommodate a protein molecule without significant changes from its natural conformation.

A qualitative estimate of adhesion was performed with the Scotch tape test. ${ }^{57}$ The tape was attached to the film and then pulled off. The $\mathrm{Ag} / \mathrm{SiO}_{2} /$ glass substrate shows good adhesion in the tape test. The changes in absorption/reflection spectra were less than 5\% after the tape test. We compare Ag substrates with and without an $\mathrm{SiO}_{2}$ sublayer and also with a Ti sublayer. Adhesion of Ag on glass (bare microscope slide) is poor, and we could not get uniform deposition and a good-quality SERS signal with Ag/glass substrates. No SERS was observed in the 
opposite case with a high-adhesion sublayer of $\mathrm{Ti}$, where protein solution deposition does not lead to spot color changes and structural modifications. In general, to get a nonadaptive film (without restructuring), either evaporation parameters (sublayer, deposition rate, increased mass thickness) or protein solution contents should be changed. It is clear that those changes can affect metal structures or protein adsorption, which makes it impossible to directly compare between SERS on adaptive and nonadaptive films. Still, our observation is that there is no detectable SERS without restructuring.

To support this conclusion, we consider FE SEM images of nanostructures outside and inside protein spots in Figure 4. The protein-mediated restructuring results in particle aggregates with smaller dielectric gaps between the neighbor particles, leading to larger SERS signals. Indeed, the aggregation of even a few particles makes a large difference for SERS enhancement (as compared to SERS from isolated particles) due to plasmon coupling and allows single-molecule detection. ${ }^{35}$ Because of a variety of particle and gap sizes, the resonant plasmon modes cover a large spectral range, with the local field being particularly large in the gaps between the particles. ${ }^{58-61}$ Experiments with cold-deposited Ag films and pyridine, reported at the early stages of SERS studies, prove that the largest enhancement occurs in interstitial regions enclosed by several silver grains. 58

The second supporting fact is the average SERS enhancement for insulin on ASF as measured in our experiments. As we show below, the macroscopic enhancement is about $3 \times 10^{6}$, which is close to the largest reported for island or percolation Ag films $\left(10^{5}\right.$ for nitrobenzonate ${ }^{23}$ and $5.3 \times 10^{5}$ for trans-1,2-bis(4pyridyl)ethylene ${ }^{43}$ ). This is also close to the theoretical limit for random metal-dielectric films, which gives about $10^{6}-10^{7}$, with a contribution of about $10^{4}-10^{5}$ from the electromagnetic enhancement and $10^{2}$ contributed from a short-range "chemical" enhancement.

It would be unlikely to obtain such a value for the SERS enhancement from a film with fixed particle positions (e.g., with very large adhesion) since it cannot be optimal for various SERS sensing of different proteins; this is in particular true for the case when interparticle distances are randomly distributed and comparable with protein size. Adaptive substrates allow nanostructure modification under protein deposition. In this way, proteins can serve as agents to provide stronger aggregation of the metal nanoparticles on the ASF, resulting in the formation of clusters of closely spaced, "protein-glued" nanoparticles, with particularly large SERS. Because the metal particles are movable, proteins placed between the particles can preserve their conformational state on the ASFs.

Our SERS spectra indicate that insulin is adsorbed on the metal surface through $\mathrm{N}$ termini. This suggests a possible scenario for insulin and silver particle interaction on the surface. Because of the specific $\mathrm{Cl}^{-1}$ adsorption, silver particles acquire excess negative charges and attract the positively charged $\mathrm{N}$-terminus. Insulin hexamers have six N-termini of B-chains exposed to the hexamer surface, three at the top and three at the bottom (see Figure 2; the hexamer is $3.5 \times 5 \mathrm{~nm}$ in size). Thus, insulin hexamers can serve as coupling agents and induce the surface restructuring within a protein spot. By this means, the addition of protein solution to the metal nanostructure can result in the structure modification that favors SERS and preserves the conformation state of proteins.

\section{SERS Spectra}

Recombinant human insulin and insulin lispro were provided by Eli Lilly \& Co. Our samples were prepared from $\mathrm{Zn}$-insulin crystals; the crystals were dissolved and aliquoted into the vials, and the vials were lyophilized. Insulin was dissolved in a $1 \mathrm{mM}$ $\mathrm{HCl}$ water solution using deionized water of ultrapure grade. Insulin concentrations were determined from absorbance measurements at $280 \mathrm{~nm}$ using the extinction coefficient $\epsilon_{280} \sim 5.7$ $\mathrm{mM}^{-1} \mathrm{~cm}^{-1}{ }^{62}$ The solution was then diluted with $\mathrm{HCl}$ solution or water. Typically, $2-4 \mu \mathrm{L}$ of $1 \mu \mathrm{M}$ insulin solution containing $0.1 \mathrm{mM} \mathrm{HCl}$ was deposited on a substrate and then allowed to dry in a $45^{\circ}$ inclined substrate position. The protein distribution in the deposited spot was obtained with an Alpha-step 200 profilometer (Tencor Instruments). The spot typically consists of an outer ring and a central part as illustrated in Figure 3. An average protein density in the spot is about $200 \mathrm{fmol} / \mathrm{mm}^{2}$ calculated from $3 \mu \mathrm{L}$ volume, $1 \mu \mathrm{M}$ concentration, and $14 \mathrm{~mm}^{2}$ area. Spot profile measurements indicate that the local density in the central portion of the spot was less than the average by a factor of 2.5. ${ }^{63}$ Finally, this estimate gives a surface density of about $80 \pm 20 \mathrm{fmol} / \mathrm{mm}^{2}$ in the central area of the spot. A typical laser beam size used in experiments was about $100 \mu \mathrm{m}$. A minimal protein amount of 25 amol in the probed area has been detected for a laser spot of about $20 \mu \mathrm{m}$ in diameter. The laser intensity of $450 \mathrm{~W} / \mathrm{cm}^{2}$ in this case is close to the upper limit for SERS spectra acquisition without incurring changes due to photomodification. The laser spot size was measured using the "knife" method.

SERS spectra were collected using a laser beam excitation wavelength of $568.2 \mathrm{~nm}$ with normal incidence and $45^{\circ}$ scattering (see details below ${ }^{64}$ ). Laser power was about $1 \mathrm{~mW}$, and the typical exposure time was 100-200 s.

Enhancement Factor. To estimate the enhancement factor, we first compare our SERS-specific intensity with the Raman signal of $\mathrm{Zn}$-insulin in liquid. ${ }^{4}$ We consider the measurement systems to be very similar in both cases in terms of collection lens $f$-number, notch filter, resolution, and CCD capabilities. We omit the possible differences caused for example by additional losses in the second notch filter and the use of a fiber bundle in our case or due to a different excitation wavelength (647.1 nm versus $568.2 \mathrm{~nm}$ for SERS). The intensity of the Raman signal, in general, is proportional to the intensity of the laser field and the number of molecules in the probed volume: $I_{\mathrm{R}} \sim I_{\mathrm{L}} N$. For the surface measurements it can be expressed through the average power $W$ and protein surface density $D$ as $I_{\mathrm{L}} N=W D$, assuming that the collection area is equal to or exceeds the illumination area. Similar considerations give the following relation for volume measurements: $I_{\mathrm{L}} N=\mathrm{WCl}$, where $C$ is concentration and $l$ is the laser beam length in the collection volume. Finally, an estimate of the enhancement is given by $G=\left(J_{\mathrm{S}} / J_{\mathrm{R}}\right)(C l / D)$, where $J_{\mathrm{S}}$ and $J_{\mathrm{R}}$ are the specific intensities (counts per second per milliwatt (counts/(s・mW)) of the 1003 $\mathrm{cm}^{-1}$ peak for both SERS and Raman. Under the conditions of ref 4 , the laser beam is imaged along the monochromator slit and the length of the beam in the collection area can be estimated as approximately $7 \mathrm{~mm}$ [J. R. Diers, private communication]. Taking into account the other values of $J_{\mathrm{R}}=$ 1 counts $/(\mathrm{s} \cdot \mathrm{mW}), C=5 \mathrm{nmol} / \mathrm{mm}^{3}(5 \mathrm{mM}), J_{\mathrm{S}}=7$ counts $(\mathrm{s} \cdot \mathrm{mW})$, and $D_{\mathrm{S}}=80 \mathrm{fmol} / \mathrm{mm}^{2}$, one can get the average enhancement of about $G=3 \times 10^{6}$.

To define the enhancement factor under the same conditions, we performed SERS and Raman measurements with the same optical system and used insulin from the same vial. ${ }^{65}$ Spectra were collected at $632 \mathrm{~nm}$ with a $\times 20$ objective lens in the backscattering configuration. In the case of normal Raman, the insulin spots were deposited on a quartz substrate at different insulin concentrations $(10-200 \mu \mathrm{M})$ and volumes in the droplet 

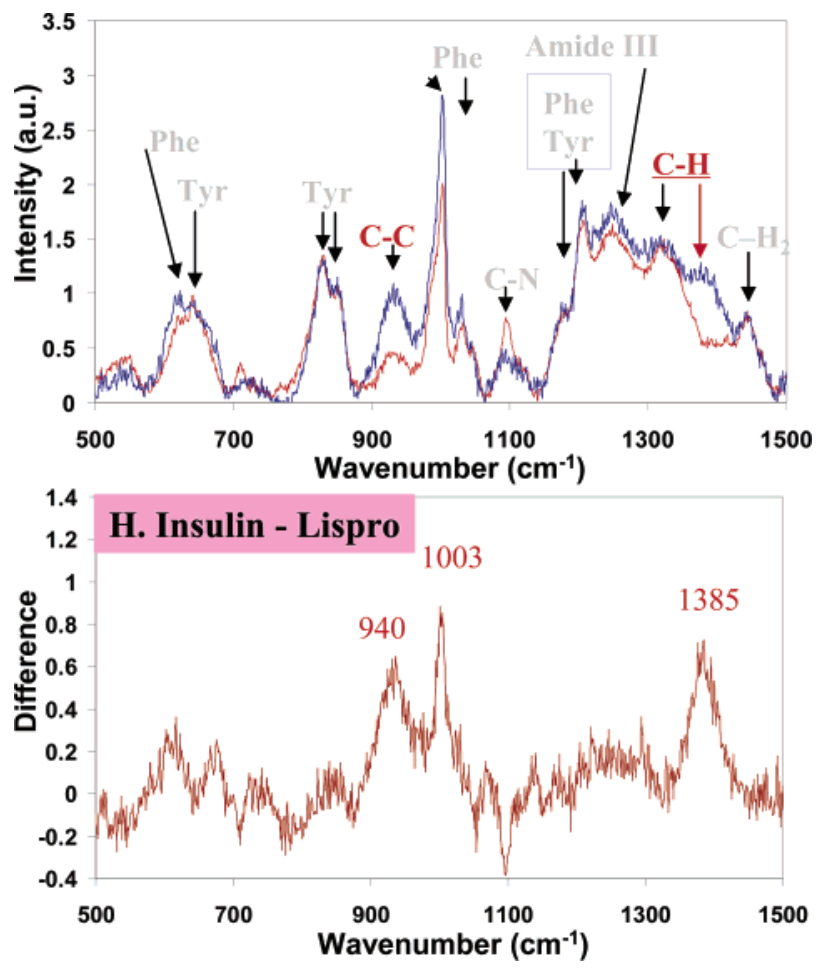

Figure 5. SERS spectra for human insulin (blue) and its analogue insulin lispro shown in red (above) and the difference SERS spectra (below).

(5-10 $\mu \mathrm{L})$ and spectra were collected from a ring of the spot. It was found that increasing the thickness of an insulin layer above $10 \mu \mathrm{m}$ does not change the signal. Using this value for the penetration depth of the collection/illumination optics, one can estimate the insulin surface density $D_{\mathrm{R}}$ as $5 \mathrm{nmol} / \mathrm{mm}^{2}$. The measured specific intensity of the Phe peak at $1003 \mathrm{~cm}^{-1}$ is about $J_{\mathrm{S}}=33$ counts $/(\mathrm{s} \cdot \mathrm{mW})$ for SERS and $J_{\mathrm{R}}=0.8$ counts/ $(\mathrm{s} \cdot \mathrm{mW})$ for Raman. Finally, the results suggest an enhancement factor $G=J_{\mathrm{S}} D_{\mathrm{R}} / J_{\mathrm{R}} D_{\mathrm{S}}$ of about $2.5 \times 10^{6}$. This is in good agreement with the previous estimate above.

Note that the local electromagnetic enhancement in the resonant nanostructures of such a semicontinuous metal film is greater than the average by several orders of magnitude, and it can reach $\sim 10^{10}$ or even larger according to theory. ${ }^{46,47}$

Representative SERS spectra collected from the central part of the spots are shown in Figure 5. The figure exhibits differences in the spectra for human insulin and insulin lispro (both spectra were subjected to linear polynomial background subtraction). The spectra are normalized to the Tyr peaks at 832 and $853 \mathrm{~cm}^{-1}$; these peaks are chosen for the normalization since they show approximately equal average intensity over three spots for both human insulin and lispro. The specific intensity, i.e., counts per second per milliwatt, was used for this comparison. Human insulin spectra, as compared to insulin lispro, indicate more intense peaks at $1387 \mathrm{~cm}^{-1}, 945 \mathrm{~cm}^{-1}$, and for the phenylalanine (Phe) ring-breathing mode at 1003 and $1030 \mathrm{~cm}^{-1}$. These differences hold at low laser intensity and power $(1 \mathrm{~mW})$ and become less pronounced under exposure to higher laser power. Figure 6 shows six spectra collected from three spots of human insulin (blue) and six spectra collected from three spots of insulin lispro (red) on the same substrate and illustrates spot-to-spot deviations in spectra.

\section{Discussion}

The zinc insulin hexamer is an allosteric protein that exhibits two different protein conformations referred to as extended (T)

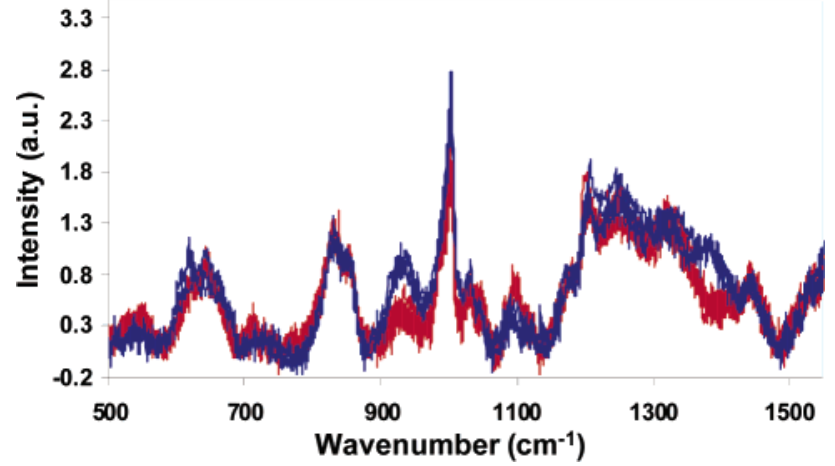

Figure 6. Human insulin and insulin lispro SERS spectra collected over three spots per each.

and $\alpha$-helical $(\mathrm{R})$ conformation states. ${ }^{66}$ The R-state conformation is observed in $\mathrm{R}_{6}$ hexamers, and it has $\mathrm{B} 1-\mathrm{B} 8$ residues in $\alpha$-helical form. Unlike the pure R state, a so-called "frayed" $\mathrm{R}^{\mathrm{f}}$ state exists in $T_{3} R_{3}$ hexamers, where only $B 4-B 8$ residues are $\alpha$-helical. In solution, $\mathrm{T}$ and $\mathrm{R}$ conformations are in equilibrium. The presence of phenol or chloride ions can shift the equilibrium toward the $\mathrm{R}$ state. The $\mathrm{T}-\mathrm{R}$ state allosteric transitions involve interconversions among three conformational states: $T_{6}, T_{3} R_{3}$, and $\mathrm{R}_{6}{ }^{66}$ The transitions are modulated by ligand binding and result in conversion of $\mathrm{B} 1-\mathrm{B} 8$ residues from a random coil to an $\alpha$-helix conformation along with about $30 \AA$ displacement of Phe B1, which can be detected by Raman spectroscopy. ${ }^{4}$

A comparison of our SERS and normal Raman spectra for insulin on a quartz surface ${ }^{65}$ and for Zn-insulin in solution ${ }^{4}$ suggests that our SERS spectra reveal all known Raman "fingerprints" of insulin. The Raman peaks are assigned mainly to amide I and amide III bands of peptide backbone vibrations, to vibrational modes of $\mathrm{Phe}(\mathrm{B} 1, \mathrm{~B} 24$, and $\mathrm{B} 25$ residues of B-chains) and tyrosine (Tyr) (A14, A19, B16, and B26). ${ }^{1}$

The SERS difference between the two insulins can be attributed, in part, to (i) Phe B1 displacement and (ii) the $\alpha$-helical N-terminus of the B-chain in human insulin, which is a feature of the R-sate conformation. As mentioned, insulin is adsorbed on the surface primarily through the N-terminus. Because of the excess negative charge, metal particles attract the positively charged $\mathrm{N}$-terminus of the $\mathrm{B}$-chain and thus move Phe(B1) closer to the surface. Depending on the conformational state of the displaced Phe(B1), the latter can be at different distances and thus have different orientations with respect to the metal surface, enabling the observed increase in Phe peak intensities by a factor of 1.4 for human insulin as compared to insulin lispro.

The $\mathrm{CH}$ deformation band at $1385 \mathrm{~cm}^{-1}$ and the $\mathrm{C}-\mathrm{C}$ skeletal band at $940 \mathrm{~cm}^{-1}$ are stronger in human insulin than in insulin lispro. The $890-945 \mathrm{~cm}^{-1}$ band is a characteristic spectral line for an $\alpha$-helix and is known to be sensitive to structural changes. ${ }^{67-70}$ This spectral line is typically centered at $940 \mathrm{~cm}^{-1}$ and disappears or displays weak intensity upon conversion to $\beta$-sheet or random coil structures. It is also known that the $\mathrm{C}-\mathrm{H}$ deformation band at $1371 \mathrm{~cm}^{-1}$ appears for the $\mathrm{R}_{6}$ conformation of hexameric human insulin, which has the longest sequence (B1-B19) of $\alpha$-helix. ${ }^{4}$ We note that this band appears in $\mathrm{R}_{6}-$ $\mathrm{T}_{6}$ Raman difference spectra and disappears in $\mathrm{T}_{3} \mathrm{R}_{3} \mathrm{f}_{6}-\mathrm{T}_{6}$ spectra. ${ }^{4}$ This points out the critical contribution of the $\alpha$-helical residues $\mathrm{Phe}(\mathrm{B} 1), \mathrm{Val}(2)$, and $\mathrm{Ala}(\mathrm{B} 3)$ at $\mathrm{N}$-termini of $\mathrm{R}_{6}$ hexamers in the $\mathrm{C}-\mathrm{H}$ deformation band at $1370-1385 \mathrm{~cm}^{-1}$. The observed differences in the SERS spectra suggest that human insulin and insulin lispro have different conformational states on the surface. Specific orientations of molecular bonds 
on the silver surface emphasize the SERS spectral difference between the two insulins, making the differences much stronger than for conventional Raman.

For both human insulin and insulin lispro, the $1 \mu \mathrm{M}$ concentration solution contained $\mathrm{Zn}$ and $\mathrm{Cl}$ ions in our experiments. The presence of chloride ions results in the $\mathrm{T} \rightarrow \mathrm{R}$ transition. Typically, it is accompanied by hexamer formation in the presence of zinc. From our data, it is difficult to define the ratio between different oligomerization states of insulin on a metal surface. However, the SERS difference spectra indicate that a preferred conformational state is the $\mathrm{R}$ state for human insulin and the $\mathrm{T}$ and/or $\mathrm{R}^{\mathrm{f}}$ state for insulin lispro. This conclusion is in agreement with $\mathrm{X}$-ray crystallographic studies, ${ }^{71}$ where it was observed that insulin lispro crystallizes as a $T_{3} R_{3}^{f_{3}}$ hexamer. Thus, the presence of zinc and chloride ions can stabilize the $\mathrm{R}_{6}$ state of human insulin hexamers, ${ }^{4}$ resulting in the observed spectral differences between human insulin and insulin lispro.

Note that protein conformation is sensitive to the environment and any kind of protein interaction with a surface. Since comparative SERS spectra reveal characteristic features of the insulin and its analogue that are correlated with normal Raman and X-ray studies, we conclude that there is no significant alteration in conformational states of insulin on the adaptive substrates.

In summary, the nanostructured adaptive silver films (ASFs) used in our experiments demonstrate their ability to preserve secondary structure of proteins and a high SERS sensitivity in detection of conformational distinctions of nearly identical proteins at the sub-monolayer level. The surface association of insulin hexamers combined with the mobility of metal particles in ASFs enables a protein-mediated aggregation of metal particles (generally resulting in increased SERS sensitivity) and establishes a platform for the observed differences in SERS spectra between human insulin and its isomer insulin lispro without denaturing the proteins. All insulin vibration modes are enhanced by approximately the same factor, which makes the SERS spectra similar to the Raman spectra in liquid and solid form and simplifies the analysis. The detected insulin surface density of about $80 \mathrm{fmol} / \mathrm{mm}^{2}$ means that one molecule is placed in the area of $4.6 \mathrm{~nm} \times 4.6 \mathrm{~nm}$. This implies a sub-monolayer coverage due to the $3.5 \times 5.5 \mathrm{~nm}$ size of insulin hexamer. The measured average enhancement factor of the SERS-active silver structure is about $3 \times 10^{6}$. The differences can be attributed in part to the $\alpha$-helical $\mathrm{N}$-terminus of the $\mathrm{B}$-chain for human insulin, which is an indicative feature for the $\mathrm{R}$ conformation state. SERS spectra were able to detect and distinguish the human insulin and insulin lispro attributes interpreted as the relative populations of $T_{3} R_{3}$ and $R_{6}$ hexamer conformations. Due to the enhanced sensitivity and spectral resolution of Raman, the ASF nanostructures are a model for SERS applications in the detection and analysis of protein structures.

Acknowledgment. We thank Drs. Ben-Amotz and Sarychev for numerous helpful discussions. This work was supported by a grant from Inproteo, LLC.

\section{References and Notes}

(1) Yu, N. T.; Liu, C. S. J. Am. Chem. Soc. 1972, 94, 3250.

(2) Yu, N. T.; Liu, C. S.; O'Shea, D. C. J. Mol. Biol. 1972, 70, 117.

(3) Yu, N. T.; Jo, B. H.; Chang, R. C. C.; Huber, J. D. Arch. Biochem. Biophys. 1974, 160, 614.

(4) Ferrari, D.; Diers, J. R.; Bocian, D. F.; Kaarsholm, N. C.; Dunn, M. F. Biopolymers (Biospectroscopy) 2001, 62, 249-260.

(5) Fleischmann, M.; Hendra, P. J.; McQuillan, A. J. Chem. Phys. Lett. $\mathbf{1 9 7 4}, 26,163$
(6) Jeanmaire, D. J.; Van Duyne, R. P. J. Electroanal. Chem. 1977, 84,1 .

(7) Albrecht, M. G.; Creighton, J. A. J. Am. Chem. Soc. 1977, 99, 5215

(8) Vo-Dinh, T. Trends Anal. Chem. 1998, 17, 557.

(9) Bauer, G.; Stich, N.; Schalkhammer, T. G. M. In Methods and Tools in Biosciences and Medicine Analytical Biotechnology; Schalkhammer, T. G. M., Ed.; Birkhauser Verlag: Basel, Switzerland, 2002.

(10) Sibbald, M. S.; Chumanov, G.; Cotton, T. M. J. Electroanal. Chem. 1997, $438,179$.

(11) Vo-Dinh, T.; Stokes, D. L.; Griffin, G. D.; Volkan, M.; Kim, U. J.; Simon, M. I. J. Raman Spectrosc. 1999, 30, 785.

(12) Brown, K. R.; Fox, A. P.; Natan, M. J. J. Am. Chem. Soc. 1996, $118,1154$.

(13) Shafer-Peltier, K. E.; Haynes, C. L.; Glucksberg, M. R.; Van Duyne,

R. P. J. Am. Chem. Soc. 2003, 125, 588.

(14) Cao, Y. W. C.; Jin, R.; Mirkin, C. A. Science 2002, 297, 1536.

(15) Cao, Y. C.; Jin, R.; Nam, J. M.; Thaxton, C. S.; Mirkin, C. A. J. Am. Chem. Soc. 2003, 125, 14676.

(16) Grubisha, D. S.; Lipert, R. J.; Park, H. Y.; Driskell, J.; Porter, M. D. Anal. Chem. 2003, 75, 5936-5943.

(17) Moskovits, M. Rev. Mod. Phys. 1985, 57, 783.

(18) Moskovits, M. J. Chem. Phys. 1978, 69, 4159.

(19) Chen, C. Y.; Burstein, E.; Lundquist, O. Solid State Commun. 1979, 32,63 .

(20) McCall, S. L.; Platzman, P. M.; Wolff, P. A. Phys. Lett. 1980, 77A, 381.

(21) Chen, C. Y.; Burstein, E. Phys. Rev. Lett. 1980, 45, 1287.

(22) Bergman, J. G.; Chemla, D. S.; Liao, P. F.; Glass, A. M.; Pinczuk,

A.; Hart, R. M.; Olson, D. H. Opt. Lett. 1981, 6, 33.

(23) Weitz, D. A.; Garoff, S.; Gramila, T. J. Opt. Lett. 1982, 7, 168

(24) Ritchie, G.; Chen, C. Y. In Surface Enhanced Raman Scattering; Chang, P. K., Furtak, T. E., Eds.; Plenum: New York, 1982; p 361.

(25) Schatz, G. C. In Fundamentals and Applications of Surface Raman Spectroscopy; Garrell, R. L., Pemberton, J. E., Cotton, T. M., Eds.; VCH: Deerfield Beach, FL, 1993.

(26) Stockman, M. I.; Shalaev, V. M.; Moskovits, M.; Botet, R.; George,

T. F. Phys. Rev. B 1992, 46, 2821.

(27) Otto, A. Surf. Sci. 1978, 75, 1392

(28) Pockrand, I.; Otto, A. Solid State Commun. 1980, 35, 861.

(29) Persson, B. N. J. Chem. Phys. Lett. 1981, 82, 56.

(30) Adrian, F. J. J. Chem. Phys. 1982, 77, 5302.

(31) Pandey, P. K.; Schatz, G. C. J. Chem. Phys. 1984, 80, 2959.

(32) Lombardi, J. R.; Birke, R. L.; Lu, T.; Xu, J. J. Chem. Phys. 1986, $84,4174$.

(33) Campion, A.; Kambhampti, P. Chem. Soc. Rev. 1998, 27, 241.

(34) Nie, S.; Emory, S. R. Science 1997, 275, 1102; J. Phys. Chem. B 1998, 102, 493 .

(35) Kneipp, K.; Wang, Y.; Kneipp, H.; Perelman, L. T.; Itzkan, I.; Dasari, R. R.; Feld, M. Phys. Rev. Lett. 1997, 78, 1667.

(36) Schatz, G. C.; Van Duyne, R. P. In Handbook of Vibrational Spectroscopy; Chalmers, J. M., Griffiths, R. P., Eds.; Wiley: New York, 2002; p 759.

(37) Musick, M. D.; Keating, C. D.; Keefe, M. H.; Natan, M. J. Chem. Mater. 1997, 9, 1499

(38) Michaels, A. M.; Nirmal, M.; Brus, L. E. J. Am. Chem. Soc. 1999 $121,9932$.

(39) Haynes, C. L.; Van Duyne, R. P. J. Phys. Chem. 2003, 107, 7426.

(40) Prodan, E.; Radloff, C.; Halas, N. J.; Nordlander, P. Science 2003, $302,419$.

(41) Davies, J. P.; Pachuta, S. J.; Cooks, R. G.; Weaver, M. J. Anal. Chem. 1986, 58, 1290 .

(42) Schlegel, V. L.; Cotton, T. M. Anal. Chem. 1991, 63, 241

(43) Van Duyne, R. P.; Hulteen, J. C.; Treichel, D. A. J. Chem. Phys. 1993, 99, 2101.

(44) Vogel, E.; Kiefer, W.; Deckert, V.; Zeisel, D. J. Raman Spectrosc. 1998, 29, 693 .

(45) Gadenne, P.; Gagnot, D.; Masson, M. Physica A 1997, 241, 161.

(46) Sarychev, A. K.; Shalaev, V. M. Phys. Rep. 2000, 335, 275.

(47) Shalaev, V. M. Nonlinear Optics of Random Media: Fractal Composites and Metal-Dielectric Films; STMP v.158; Springer: Heidelberg, Germany, 2000.

(48) Johnson, P. B.; Christy, R. W. Phys. Rev. B 1972, 6, 4370

(49) Kittel, C. Introduction to Solid State Physics; Wiley: New York, 1995.

(50) Holt, R. E.; Cotton, T. M. J. Am. Chem. Soc. 1989, 111, 2815.

(51) Yang, M.; Chang, F. L.; Thompson, M. Anal. Chem. 1993, 65, 3713.

(52) Reipa, V.; Gaigalas, A.; Abramovitz, S. J. Electroanal. Chem. 1993, 348,413 ,

(53) Callender, R.; Deng, H.; Gilmanshin, R. J. Raman Spectrosc. 1998, $29,15$.

(54) Rahuel-Clermont, S.; French, C. A.; Chou, C. I.; Kaarsholom, N. C.; Dunn, M. F. Biochemistry 1997, 36, 5837. 
(55) We fabricated our ASFs using the following procedure. Microscope glass slides (from Fisher) were cut into $2.5 \mathrm{~cm} \times 2.5 \mathrm{~cm}$ sections for use as initial substrates. The cut slides were cleaned through several steps, including multiple solvent rinses, a piranha $\left(1: 3 \mathrm{H}_{2} \mathrm{O}_{2}: \mathrm{H}_{2} \mathrm{SO}_{4}\right)$ acid bath, rinsing in $18 \mathrm{M} \Omega$ deionized water, and drying with pressurized gaseous nitrogen. Silver shot from Alfa Aesar (99.9999\%, 1-3 mm) was used for fabrication of the ASFs on the glass substrates. The thin film deposition was performed in a modified Leybold electron-beam evaporator with an initial pressure inside the system of approximately $10^{-7}$ Torr. The film thickness and deposition rate were monitored with a quartz crystal oscillator. The glass slides were covered first by a sublayer of $\mathrm{SiO}_{2}(10 \mathrm{~nm})$ followed by an Ag layer $(8 \mathrm{~nm})$ deposited at a rate of $0.05 \mathrm{~nm} / \mathrm{s}$. The fabricated films were characterized by field emission scanning electron microscopy (FE SEM), atomic force microscopy (AFM), and a Lambda 35 spectrophotometer (Perkin-Elmer) equipped with a Labsphere. High-resolution FE SEM images were obtained through MAS Inc. (Raleigh, NC). AFM images were acquired with a Dimension 3100 (DI Veeco) using a $10 \mathrm{~nm}$ Si tip for measuring height profiles of our samples.

(56) This experiment emphasizes the importance of chemical content versus surface tension of drying protein droplets in restructuring at the nanoscale. Note that proteins of other kinds may cause restructuring even in water solution.

(57) Eckertova, L. Physics of Thin Films; Plenum Press: New York and London, 1977.

(58) Albano, E. V.; Daiser, S.; Ertl, G.; Miranda, R.; Wandelt, K. Phys. Rev. Lett. 1983, 51, 2314.

(59) Krenn, J. R.; et al. Phys. Rev. Lett. 1999, 82, 2590.

(60) Quinten, M. Appl. Phys. B 2001, 73, 245.

(61) Genov, D. A.; Sarychev, A. K.; Shalaev, V. M.; Wei, A. Nano Lett. 2004, 4, NL-0343710.
(62) Porter, R. R. Biochem. J. 1953, 53, 320

(63) A profilometer stylus may scratch soft material, which results in poor accuracy in profile measurements. To avoid this problem, the lowest possible stylus force $(5 \mathrm{mg})$ was applied to our samples. We were able to detect a profile contrast of about 10 for insulin spots. A representative profile is shown in ref 65 . Since an area of the spot ring is relatively small, an estimate for the profile contrast in the range 5-12 gives good enough accuracy for the ratio between the local protein density in the central part and average one, which is about $0.4 \pm 0.1$.

(64) Our four-wavelength Raman system comprises an $\mathrm{Ar} / \mathrm{Kr}$ ion laser (Melles Griot), a laser band-path holographic filter (to reject plasma lines), two Super-Notch Plus filters to reject Rayleigh scattering (Kaiser Optical Systems), focusing and collection lenses, an Acton Research 300i monochromator with a grating of 1200 grooves/mm, and a nitrogen-cooled CCD $(1340 \times 400$ pixels, Roper Scientific). An objective lens (f/1.6) provides a collection area of about $180 \mathrm{um}^{2}$. Collected light is delivered to the monochromator via a fiber bundle. The spectral resolution is about $3 \mathrm{~cm}^{-1}$.

(65) Drachev, V. P.; Thoreson, M. D.; Khaliullin, E. N.; Sarychev, A K.; Zhang, D.; Ben-Amotz, D.; Shalaev, V. M. SPIE Proc. 2003, 5221, 76

(66) Kaarsholm, N. C.; Ko, H. C.; Dunn, M. F. Biochemistry 1989, 28 4427.

(67) Yu, T. J.; Lippert, J. L.; Peticolas, W. L. Biopolymers 1973, 12, 2161.

(68) Chen, M. C.; Lord, R. C.; Mendelson, R. Biochim. Biophys. Acta $1973,328,252$.

(69) Frushour, B. G.; Koenig, J. L. Biopolymers 1974, 13, 1809.

(70) Chen, M. C.; Lord, R. C.; Mendelson, R. J. Am. Chem. Soc. 1976 96, 3038.

(71) Ciszak, E.; Beals, J. M.; Frank, B. H.; Baker, J. C.; Carter, N. D.; Smith, G. D. Structure 1995, 3, 615 